association between HIV prevalence and physical violence was 1.59 (95\% confidence interval [95\% CI]: 1.01-2.51). The corresponding AORs for sexual and psychological violence were 1.67 (95\% CI: 1.02-2.72) and 1.60 (95\% CI: 1.10-2.33), respectively. Exposure to a larger number of violence types was associated with a progressively increasing HIV prevalence: $\mathrm{AOR}=1.39$ for one type of violence, $\mathrm{AOR}=1.75$ for two types and $\mathrm{AOR}=2.43$ for three types $(p=0.005$, test for trend).

Conclusions This study shows an association between exposure to three different forms of violence and HIV prevalence among FSWs in Benin. Although violence could also be a consequence of an HIV-positive status, it is also likely to be a distal determinant of HIV acquisition. Interventions are needed to reduce violence towards FSWs; this should be integrated into HIV prevention programmes.

\section{P3.111 THE PREVALENCE OF HIV IN MALE SEX WORKERS IN LONDON (2002 - 2012)}

doi:10.1136/sextrans-2013-051184.0570

L Mulka, R Malek, G King, D Wilkinson. Imperial College Healthcare NHS Trust, London, UK

Background The prevalence of HIV in the UK is increasing steadily, particularly in higher-risk populations. Male sex-workers (MSW) are a particularly vulnerable group, often engaging in highrisk sexual activities with multiple partners, with increased barriers to accessing care. The objective of this study was to determine how the prevalence of HIV in patients attending a specialist MSW clinic in London has changed over the last ten years.

Methods Total attendances of MSW attending a dedicated clinic in Central London were collected, and records reviewed for two time periods (1/1/2002-31/12/2012 and 1/1/2012-31/12/2012). HIV status of MSW was determined by attendance at the HIV unit at the Central London Hospital. Details of CD4 count, HAART, viral load (VL), sexual practises and condom use were obtained and compared. Results 292 men attended the clinic in 2002 and 257 in 2012 $5(1.7 \%)$ were known HIV positive in 2002, 33 (12.8\%) in 2012. In 2002, 1 patient $(20 \%)$ was on HAART with an undetectable VL and 4 had CD4 counts above treatment threshold. In 2012, 18 patients $(55 \%)$ were on HAART, 12 of which ( $67 \%$ of total) had an undetectable VL. 12 patients (36\%) were not on HAART and had a CD4 count of $>350.3$ transferred care.

Conclusions There has been a dramatic increase in the prevalence of HIV in MSW attending a dedicated clinic in London over the last decade $(1.7 \%$ in $2002,12.8 \%$ in 2012$)$. This may be due, in part, to increased uptake through the introduction of 'opt-out' testing for HIV in GUM clinics in recent years. This increasing prevalence of HIV reflects the high-risk status of MSW highlighting the importance of specialised clinics providing risk reduction strategies such as promotion of condom use, regular STI screening, post-exposure prophylaxis and HAART to reduce outwards transmission in this cohort.

\section{P3.112 HIGH PREVALENCE OF GONORRHOEA AND HPV AMONG MALE SEX WORKERS IN THREE CITIES OF VIETNAM: CHALLENGES IN ADDRESSING HIV EPIDEMIC AMONG MSM POPULATIONS}

doi:10.1136/sextrans-2013-051184.0571

'V D Vu, ${ }^{2} \mathrm{G}$ M Le, ${ }^{1} \mathrm{~S} M$ Nguyen, ${ }^{3} \mathrm{M}$ C Clatts, ${ }^{4} \mathrm{~L}$ A Goldsamt. ' Center for Research and Training on HIVIAIDS, Hanoi Medical University, Hanoi, Viet Nam; ${ }^{2}$ Department of

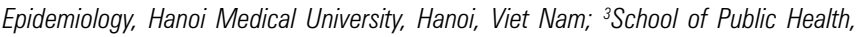
University of Puerto Rico, San Juan, Puerto Rico; ${ }^{4}$ National Development and Research Institutes, Inc., New York, NY, United States

Background MSM populations in Vietnam are faced with a rapidly growing HIV epidemic, yet little is known about STIs epidemic in this diverse population. This study describes prevalence of Gonorrhea, HPV among male sex workers, and key correlates in three major cities.

Method Cross-sectional surveys from 2009 to 2011 used TimeLocation-Sampling to recruit eligible participants. Eligibility criteria included being biological male at birth; self-report of having sex with a male partner in exchange for material rewards within the last 90 days; and age from 16 to 35. Blood samples were taken for HIV testing; pharyngeal, anal and urethral swabs for gonorrhoea and HPV.

Results Of 710 participants, $4.2 \%$ was HIV positive. Testing for gonorrhoea and HPV showed high prevalence of $28.8 \%$ and $33.2 \%$ respectively. Pharyngeal test for gonorrhoea $(23.7 \%)$ and anal test for HPV $(26.1 \%)$ were highest among swapping sites. The rates of infection were particularly higher in Ho Chi Minh City (largest economic city) as compared to Hanoi (political capital) and Nha Trang (major beach city). Odds ratio controlling for cities showed that testing positive for gonorrhoea was associated with engaging in oral sex past 30 days $\left(\mathrm{OR}^{\mathrm{M}-\mathrm{H}}=4.33\right.$; $\left.\mathrm{CI}=1.3-14.4\right)$; having receptive anal sex with more than three clients past 30 days $\left(O R^{\mathrm{M}-\mathrm{H}}=2.03\right.$; $\mathrm{CI}=1.18-3.51)$. HPV infection was associated with engaging in sex work for more than two years $\left(\mathrm{OR}^{\mathrm{M}-\mathrm{H}}=1.6\right.$; $\left.\mathrm{CI}=1.09-2.34\right)$; having receptive anal sex past 30 days $\left(\mathrm{OR}^{\mathrm{M}-\mathrm{H}}=1.95 ; \mathrm{CI}=1.35-2.83\right)$; having oral sex with more than four clients past 30 days $\left(\mathrm{OR}^{\mathrm{M}-\mathrm{H}}=1.63 ; \mathrm{CI}=1.12-2.39\right)$.

Conclusion The unprecedented high prevalence of pharyngeal Gonorrhea and anal HPV among MSW is significant given the high HIV prevalence among a relatively young population. Appropriate STIs is important in addressing the twin epidemics among MSM in Vietnam.

\section{P3.113 MALE SEX WORKERS HAVING SEX WITH MEN (MSW) IN THE NETHERLANDS: A HIDDEN POPULATION, AT A HIGHER RISK FOR STI/HIV THAN FSW AND MSM}

doi:10.1136/sextrans-2013-051184.0572

${ }^{1}$ A Haasnoot, ${ }^{1,2} \mathrm{~N}$ H T M Dukers-Muijrers, ${ }^{1} \mathrm{R}$ J H Keesmekers, ${ }^{1} \mathrm{D}$ M J Telg, ${ }^{1,2} \mathrm{~A}$ M Niekamp, ${ }^{1,2} \mathrm{C} \mathrm{J} \mathrm{P} \mathrm{A} \mathrm{Hoebe.} \mathrm{'Department} \mathrm{of} \mathrm{Sexual} \mathrm{Health,} \mathrm{Infectious} \mathrm{Diseases} \mathrm{and}$ Environment, South Limburg Public Health Service, Geleen, The Netherlands; ${ }^{2}$ Department of Medical Microbiology, School of Public Health and Primary Care (CAPHRI), Maastricht University Medical Center (MUMC+), Maastricht, The Netherlands

Background During outreach activities of our public health STIclinic a group of young men was encountered, who engaged in internet escort activities. Their commercial sex activities with men took place hidden from public sight and regular health care. This study aims to describe the STI incidence and risk behaviour in these male sex workers having sex with men (MSW), compared to female sex workers (FSW) and men who have sex with men (MSM) attending the same STI-clinic.

Methods Retrospective cross-sectional study among self-identified MSW, FSW and MSM at our STI-clinic in the Netherlands (January 2009-May 2012). All clients completed a questionnaire on sexual behaviour and were tested for STI: at multiple anatomic sites (anal, genital, oral) for chlamydia and gonorrhoea, and in serum for hiv, hepatitis B and syphilis. Clinical consultations $(n=3716)$ from MSW (n=203), FSW ( $=801)$ and MSM $(n=2712)$ were compared using chi-square statistics.

Results A new STI was diagnosed in $42 \%$ of MSW; this proportion was lower in MSM (14\%; p < 0.01) and FSW (9\%; p < 0.01). Of MSW 8\% showed a new hiv-infection $(0 \%$ in FSW; $\mathrm{p}<0.01$ and $1 \%$ in MSM; $p=0.03)$.

Majority (87\%) of MSW originated from Eastern Europe, their median age was 24 years. Less than half of the men self-identified as homosexual, and indeed $58 \%$ also reported sex with women (28\% of MSM; $p$ < 0.01). MSW reported sex contacts with (other) sex 